\title{
Potential Therapeutic Effects of Psilocybin
}

\author{
Matthew W. Johnson ${ }^{1} \cdot$ Roland R. Griffiths ${ }^{1,2}$
}

Published online: 5 June 2017

(C) The American Society for Experimental NeuroTherapeutics, Inc. 2017

\begin{abstract}
Psilocybin and other 5-hydroxytryptamine $2 \mathrm{~A}$ agonist classic psychedelics have been used for centuries as sacraments within indigenous cultures. In the mid-twentieth century they were a focus within psychiatry as both probes of brain function and experimental therapeutics. By the late 1960s and early 1970s these scientific inquires fell out of favor because classic psychedelics were being used outside of medical research and in association with the emerging counter culture. However, in the twenty-first century, scientific interest in classic psychedelics has returned and grown as a result of several promising studies, validating earlier research. Here, we review therapeutic research on psilocybin, the classic psychedelic that has been the focus of most recent research. For mood and anxiety disorders, three controlled trials have suggested that psilocybin may decrease symptoms of depression and anxiety in the context of cancer-related psychiatric distress for at least 6 months following a single acute administration. A small, open-label study in patients with treatmentresistant depression showed reductions in depression and anxiety symptoms 3 months after two acute doses. For addiction, small, open-label pilot studies have shown promising success rates for both tobacco and alcohol addiction. Safety data from these various trials, which involve careful screening, preparation, monitoring, and follow-up, indicate the absence of severe
\end{abstract}

Matthew W. Johnson

mwj@jhu.edu

1 Department of Psychiatry and Behavioral Sciences, Johns Hopkins University School of Medicine, Baltimore, MD, USA

2 Department of Neuroscience, Johns Hopkins University School of Medicine, Baltimore, MD, USA drug-related adverse reactions. Modest drug-related adverse effects at the time of medication administration are readily managed. US federal funding has yet to support therapeutic psilocybin research, although such support will be important to thoroughly investigate efficacy, safety, and therapeutic mechanisms.

Key Words Psilocybin · psychedelic · cancer · addiction · depression $\cdot$ anxiety

\section{Introduction}

Various indigenous societies have used classic psychedelic compounds (e.g., psilocybin, mescaline, dimethyltryptamine) for centuries, typically incorporating them into sacramental contexts [1-3]. Arthur Heffter isolated mescaline, the principal psychoactive constituent of the peyote cactus, in 1897 [4, 5], but it was only after the synthesis and identification of psychoactivity of lysergic acid diethylamide (LSD) in 1943 [6] that these compounds received significant attention from the scientific community [7]. There were $>1000$ clinical papers published on classic psychedelics, collectively involving approximately 40,000 patients between 1950 and the mid-1960s [8]. Aside from basic research studies, this period generated exciting preliminary evidence that classic psychedelics, when administered in the context of psychotherapy, showed particular promise for 2 disorders: end-of-life psychiatric distress secondary to cancer [9-21] and addiction [22-29]. While some initial results were promising, most studies did not employ rigorous designs by current standards. Nonmedical classic psychedelic use, and the association between classic psychedelics and the emerging counterculture, prompted a political backlash whereby human classic psychedelic research was marginalized, government funding for classic 
psychedelic research ended, and regulations made such research difficult, putting an end to nearly all human classic psychedelic research. Modern research with classic psychedelics has reinitiated interest in the treatment of both cancer-related distress and addiction, with promising initial results. Although LSD was the most studied classic psychedelic compound in the earlier era of research, recent clinical research has been primarily focused on the classic psychedelic psilocybin, which is closely pharmacologically related to LSD. Here we focus on these two potential indications for psilocybin medication development.

\section{Psilocybin}

Over 100 species of mushrooms have been found to contain psilocybin, many falling within the genus Psilocybe [30]. Psilocybin is a relatively small compound based on the structure of tryptamine. Psilocybin is a prodrug metabolized through in vivo dephosphorylation to psilocin, which is presumed to be the active agent in the central nervous system [31]. Like other classic psychedelics, the behavioral effects of psilocybin appear to be mediated primarily by agonist activity at the 5-hydroxytryptamine $(\mathrm{HT})_{2 \mathrm{~A}}$ receptor; however, $5-\mathrm{HT}_{2 \mathrm{~A}}$ activity does not appear to account fully for its effects [32]. Psilocybin is characterized by low physiological toxicity [33] and low abuse liability, as demonstrated by marginal levels of nonhuman drug self-administration [34].

\section{Treatment Model}

For the therapeutic laboratory studies of psilocybin to be described later in this review, psilocybin was administered as part of structured psychotherapy, unlike most psychiatric medications. This therapeutic context includes screening to exclude those with psychotic disorders, preparation of the participant for sessions, session monitoring and interpersonal support that encourages internal focus, and postsession discussion of session experiences to leverage those experiences in encouraging enduring changes in behavior and attitude. These factors are believed to maximize efficacy while minimizing psychiatric adverse effects. Individuals with current psychosis or who are at risk for psychotic disorders are thought to be at increased risk for prolonged psychiatric reactions resulting from classic psychedelic administration, and are therefore excluded from psilocybin studies. Psilocybin moderately increases blood pressure [35], so those at high risk for cardiovascular problems are also excluded. Aside from adverse reactions that could occur if these exclusions were not made, the major risk of administering psilocybin and other classic psychedelics is that they can cause psychologically overwhelming anxious, fearful, and confused reactions that can potentially lead to dangerous behavior in unmonitored settings.
Participant preparation, session monitoring, and follow-up discussion about sessions all are thought to minimize the occurrences of such adverse reactions [36]. Although psilocybin has been shown to cause dose-related increases in transient postsession headaches, this does not constitute a contraindication for therapeutic studies [37]. For more detailed information on the safety profile of psilocybin discussed above, as well as recommended guidelines for safely conducting such sessions, see a previous publication by the present authors [37].

\section{Cancer-Related Psychiatric Distress}

A small double-blind, placebo-controlled, cross-over study administered a moderate dose of oral psilocybin $(0.2 \mathrm{mg} / \mathrm{kg}$ oral $)$ and active placebo (niacin) in separate sessions ("several weeks apart") to 12 patients with advanced-stage cancer [38]. These patients were diagnosed with one of multiple possible DSM-IV anxiety-related disorders in relation to their cancer. Although the size was small, the study showed nonsignificant trends for psilocybin to decrease depression [Beck Depression Inventory (BDI)] and anxiety [State-Trait Anxiety Inventory (STAI)] more than placebo in the 2 weeks following each of the two conditions. Moreover, compared with the time of baseline screening, anxiety was reduced at a 3-month follow-up and depression outcomes were significantly reduced at a 6-month follow-up. Psilocybin caused no clinically significant adverse events.

A recent study published by the present authors examined a larger dose of psilocybin in the treatment of depression and anxiety in patients with cancer [39]. Patients were 51 individuals diagnosed with one of multiple possible DSM-IV moodor anxiety-related disorders in relation to a life-threatening cancer diagnosis. This study used a double-blind crossover design to compare oral psilocybin $(\sim 0.31$ or $\sim 0.43 \mathrm{mg} / \mathrm{kg}$ ) with a very low dose of psilocybin $(\sim 0.014$ or $\sim 0.043 \mathrm{mg} /$ $\mathrm{kg}$ ) that was expected to have little pharmacological activity. The two sessions were separated by approximately 5 weeks. The study showed the high psilocybin dose to result in numerous improved clinical outcomes over the very-low-dose condition at 5 weeks (before the crossover). Moreover, the Hamilton Anxiety Rating Scale, the STAI, the Hamilton Depression Rating Scale, and the BDI all showed large and persisting reductions at a 6-month follow-up. These results are remarkable not only because they show persistent benefits for many months after a single medication administration, but also because of the large magnitude of clinical effects. Approximately $80 \%$ of participants at the 6-month follow-up continued to show clinically significant decreases in depressed mood and anxiety, and approximately $60 \%$ showed remission: in other words, symptom levels in the normal range. Like previous research in healthy volunteers [35], long-term benefits were greater for those whose psilocybin session subjective 
experiences were high in the construct of mystical-type experience, which involves the following psychological qualities: a sense of unity, a noetic quality, sacredness, positive mood, transcendence of time and space, and ineffability. No serious adverse events were attributed to psilocybin administration.

Another recent study using a similar double-blind crossover design examined 29 patients with cancer diagnosed with one of several DSM-IV anxiety-related disorders [40]. Patients were administered a moderate oral dose of $0.3 \mathrm{mg} / \mathrm{kg}$ oral psilocybin and active placebo (niacin) in different sessions separated by approximately 7 weeks. Like the previously described study [39], this study found significant clinical improvements in numerous domains when comparing the two conditions before the crossover, including reduced anxiety and depression, and increased quality of life. At a follow up at about 6 months, the study showed enduring anxiolytic and antidepressant effects compared with baseline measures, using measures that largely overlapped with the previously described study [39]. Like that study, clinical response was large, with antidepressant and anxiolytic response rates of approximately $60 \%$ to $80 \%$ at the 6month follow up, and reductions in anxiety and depression were greater for those patients who had subjective mystical-type experiences during psilocybin sessions. Also like the other studies in cancer patients, no serious adverse events were attributed to psilocybin administration.

Although not conducted with psilocybin, another recent blinded study using the related compound LSD lends support to the abovementioned psilocybin results [41]. Twelve patients with anxiety associated with life-threatening diseases (6 with cancer) were randomized to be treated with either $200 \mu \mathrm{g}$ LSD or a very low dose of $20 \mu \mathrm{g}$ LSD intended to serve as an active placebo. The study showed nonsignificant reduction in anxiety using the STAI, which persisted for 12 months after treatment. As with the psilocybin studies, there were no serious adverse effects resulting from LSD administration.

Although the original era of classic psychedelic research focused on end-of-life cancer-related distress [9-21], some of the promising recent studies discussed above included some participants who did not have a terminal diagnosis [39, 40], and other recent research has included some participants with a life-threatening disease other than cancer [41]. Classic psychedelics might therefore hold potential therapeutic efficacy for psychiatric distress across both terminal and nonterminal cases of cancer, as well as across both cancer and noncancer disease states. Future research should investigate potential differences in efficacy across these domains.

\section{Treatment-Resistant Depression}

Outside of the context of cancer, a recent small, open-label, pilot study tested the effect of psilocybin in treatment-resistant major depression [42]. Patients received $10 \mathrm{mg}$ oral psilocybin on the first session, and $25 \mathrm{mg}$ in a second session 1 week later. Depressive symptoms, as measured by the Quick Inventory of Depressive Symptoms, the BDI, and other measures, were significantly decreased at 1 week and 3 months post-treatment, when compared with baseline scores. The same pattern was found for anxiety symptoms as measured by the STAI. Every individual participant showed reduction in depression severity at 1 week that was sustained in the majority for 3 months. According to standard criteria for determining remission with the BDI, 8 of 12 met threshold for complete remission, and 5 of 12 were in remission at the final 3month follow-up. Psilocybin did not cause any unexpected or serious adverse events.

\section{Addiction}

Research examining the potential of classic psychedelics to treat addiction occurred as early as the 1950s [22]. These investigators initially thought classic psychedelics might safely mimic the effects of delirium tremens and these aversive effects would prompt sobriety among alcoholics. However, they soon realized that insightful effects often occurred and were claimed to occasion sobriety. Such effects prompted one of these early researchers, Humphry Osmond, to coin the term "psychedelic", meaning "mind-manifesting" as a descriptor of this class of drugs [43]. Some studies examining classic psychedelics in the treatment of alcoholism did not contain experimental elements critical for confirming causal efficacy such as randomization and comparison conditions, and other small randomized studies found improved outcomes for the classic psychedelic group that were not statistically significant. Firm conclusions from this literature were unclear. However, a recent meta-analysis examining the only 6 studies to randomize participants to LSD or another condition (total participants $=536$ ) found overall significant decreases in alcohol misuse in the LSD groups compared with control groups, with an odds ratio for improvement with LSD of 1.96 at the initial follow-up, which was at least 1 month after treatment [44]. Notably, every one of the original six studies showed an odds ratio that favored LSD. In addition to these early medical studies suggesting potential efficacy of classic psychedelics in addictions treatment, a number of observational studies documented reports of recovery from addiction to alcohol and other drugs resulting from ceremonies involving classic psychedelic consumption among religions that view such compounds as sacraments [45-56]. Consistent with medical and observational studies, the founder of Alcoholic Anonymous, Bill Wilson, was a proponent of the theoretic potential of classic psychedelics to facilitate treatment of addiction. Wilson concluded this after receiving medically supervised LSD sessions, and likening the effects to the spiritual experience that had prompted his sobriety decades beforehand [57]. 
Recent studies examining the classic psychedelic psilocybin in the treatment of addiction are currently at a less advanced stage of research than the studies on cancer-related psychiatric distress reviewed earlier. These studies were conducted with relatively limited resources and were designed to establish safety and determine if efficacy results are encouraging enough to warrant the effort and funding required to conduct larger randomized clinical trials.

The present authors conducted an open-label pilot study that administered psilocybin to 15 treatment-resistant tobacco/nicotine-dependent smokers, in the context of cognitive behavioral therapy for smoking cessation [58]. Participants were administered $\sim 0.29 \mathrm{mg} / \mathrm{kg}$ oral psilocybin on the participant's target date to quit smoking, which was assigned several weeks ahead of time. A second and third (optional) dose of psilocybin were administered 2 and 8 weeks, respectively, after the target quit date. In these subsequent sessions the dose administered was increased to $\sim 0.43$ $\mathrm{mg} / \mathrm{kg}$ unless researchers and participants decided to remain at the original dose based on the first session experience. Weekly cognitive behavioral therapy sessions were conducted up to 10 weeks after target quit date except when a psilocybin session was scheduled. At the 6-month follow up, 12 of 15 participants (80\%) were abstinent from smoking based on biological verification with breath carbon monoxide and urine cotinine results. At a 12-month follow-up, 10 of 15 participants (67\%) were biologically verified as abstinent [59]. At a long-term term follow that averaged 2.5 years after the target quit date, 9 of the 12 participants $(75 \%)$ were biologically verified as abstinent [59]. No serious adverse events were caused by psilocybin. The results of this open-label pilot study cannot be taken as solid evidence for the efficacy of psilocybin for smoking cessation. However, the high rates of abstinence compared with existing medications provide justification for a larger randomized trial the present authors are currently conducting.

Regarding psychological mechanisms or correlates of efficacy, those participants in the smoking cessation pilot study who were abstinent at the 6-month follow-up had significantly higher scores on a measure of mystical-type experience resulting from psilocybin compared with those who had relapsed to smoking [60]. Moreover, higher mystical-type experience scores significantly correlated with a greater reduction in craving from baseline to 6-month follow-up [60]. Consistent with results for cancer-related psychiatric distress reviewed above, these results suggest that specific types of psychological experiences, not just receiving the medication, are important for beneficial therapeutic outcomes.

Another clue regarding psychological mechanisms underlying psilocybin effects in smoking cessation is provided by a recent online survey study conducted by the present authors examining 358 individuals reporting to have quit or reduced smoking after having taken a classic psychedelic [61].
Specifically, participants reported less severe affective withdrawal symptoms (e.g., depression, craving) when compared with their previous attempts without classic psychedelics. This provides another potential connection to the cancer-related psychiatric distress and treatment resistant depression research. That is, affective processes might play a key role in therapeutic psilocybin effects, even outside of the context of mood disorders.

Similar to the smoking cessation pilot study described above, another small, open-label study examined psilocybin in the treatment of alcohol dependence [62]. Ten alcoholdependent participants were administered psilocybin in the context of a 12-week program of motivational enhancement therapy. Participants had to have been abstinent from alcohol for $24 \mathrm{~h}$ before their first psilocybin session, in which $0.3 \mathrm{mg} /$ $\mathrm{kg}$ oral psilocybin was administered. For some participants a second session occurred 4 weeks later, in which $0.4 \mathrm{mg} / \mathrm{kg}$ was administered, although the lower previous dose was sometimes administered based on the effects of the initial session. Four therapy sessions were conducted between the two psilocybin sessions, and an additional four therapy sessions were conducted after the final psilocybin session. Mean selfreported alcohol use decreased significantly after the first psilocybin session, and remained lower than baseline at 36-week follow-up. Drinking was reported to have occurred on $40 \%$ of days at baseline, and on $<20 \%$ of days at follow up. Consistent with findings from the studies of cancer-related psychiatric distress and smoking cessation, higher mysticaltype experience scores in the first psilocybin session were significantly related to greater reductions in alcohol consumption. No serious adverse events were reported as a result of psilocybin administration. Encouraged by these promising signs of potential efficacy, this research group has initiated a large randomized trial of psilocybin in the treatment of alcohol dependence

\section{Other Disorders}

\section{Obsessive-Compulsive Disorder}

One pilot study in 9 participants examined the effect of oral psilocybin in patients with obsessive-compulsive disorder [63]. Doses administered were $0.025,0.1,0.2$, and $0.3 \mathrm{mg} /$ $\mathrm{kg}$. A week elapsed between each session. All participants showed substantial symptom reduction during at least one session as assessed by the Yale-Brown Obsessive Compulsive Scale. At a 6-month follow up, 1 participant showed longterm improvements. Although these results might suggest psilocybin efficacy, it should be noted that a similar magnitude of symptom reduction was observed at all dose conditions, including the extremely small dose intended to have little or no effect. The similar response across such a wide range of doses 
raises the possibility that placebo (e.g., expectancy) effects may have driven results. Otherwise, the symptom reductions at the lowest dose would appear remarkable. Only follow-up experimental studies involving a placebo or active comparator control will be able to disentangle these two possibilities.

\section{Cluster Headaches}

One published case series of 53 self-medicating patients suggested that psilocybin-containing mushrooms, in addition to LSD, may be effective in terminating cluster headaches or preventing the regular occurrence of cluster headaches [64]. This is exciting because approved therapies show limited efficacy in treating this disorder, and the pain resulting from the disorder is often severe and debilitating. Interestingly, case reports suggest efficacy with doses that do not result in noticeable psychoactive effects [64]. If rigorous clinical trials confirm these results, this therapeutic application would likely be distinct from the other classic psychedelic therapies, in that positive therapeutic outcomes do not appear to rely on subjective experiences after psilocybin administration. In addition to the case series publication, a survey study of 496 individuals with cluster headaches suggested that psilocybin and related classic psychedelics may provide comparable or better treatment responses than existing approved therapies [65]. Although potential efficacy of psilocybin for cluster headaches is exciting, it is important to keep in mind that evidence to date is based on self-medicating individuals using nonregulated substances, and involves no control for placebo effects. Also, it should be noted that an open-label pilot study of a non-psychoactive compound (2-bromo-LSD) with structural similarities to LSD showed promising effects [66]. If future research confirms that this or other nonpsychoactive analogs of classic psychedelics provide relief from cluster headaches, it would likely decrease the chances that psilocybin would be developed into an approved medicine for this indication, given that potential psychoactive effects would be considered unnecessary side effects.

\section{Biological Mechanisms}

Considerable information is known about mechanisms underlying the general activity of psilocybin and other classic psychedelics, including a primary role of $5-\mathrm{HT}_{2 \mathrm{~A}}$ agonist action for psychoactive effects [31]. However, no study investigating potential therapeutic uses of psilocybin has identified biological mechanisms that distinguish successful versus nonsuccessful therapeutic outcomes, which could suggest mechanisms responsible for therapeutic efficacy. The most promising line of research may be resting-state function magnetic resonance imaging analyses showing psilocybin administration to alter acutely brain network activity, including decreasing connectivity within the default mode network, a system of brain regions that strongly interact with each other, and which supports internal focus [67]. A challenge to biological theories of therapeutic classic psychedelic effects is the fact that therapeutic effects persist long after acute drug effects. One plausible hypothesis is that the acute destabilization of brain networks by psilocybin (which may stem from receptor level effects via amplification of neuronal avalanches) may provide the opportunity to alter brain network activity in a persisting fashion [68]. Aside from addressing the persisting nature of therapeutic effects, this hypothesis is also attractive because it may account for the strong role that appropriate context ("set and setting") and psychotherapy are thought to play in therapeutic classic psychedelic effects [36]. That is, classic psychedelic administration may occasion a plastic state with regard to brain network activity, and the psychotherapeutic context surrounding classic psychedelic administration (which includes preparation for sessions, session monitoring and interpersonal support that encourages internal focus, and postsession discussion of session experiences to leverage those experiences in encouraging enduring changes in behavior and attitude change) may serve to establish longer-term changes in brain network activity. It remains for future research to test this hypothesis.

\section{Conclusion}

The current state of modern research suggests considerable therapeutic promise for psilocybin. This research is most advanced regarding the treatment of cancer-related psychiatric distress, with three randomized, placebo-controlled trials showing promising results for psilocybin [38-40]. Two of these trials involved a moderate number of participants and administered relatively large doses of psilocybin [39, 40]. These two studies, in particular, provide strong evidence showing substantial decreases in depressive and anxious symptoms that appear to persist for at least 6 months after a single active treatment. Such results are unprecedented in psychiatry. The single open-label study of treatment-resistant depression outside the context of cancer provides initial preliminary evidence that persisting antidepressant effects of psilocybin might not be limited to those with cancer [42]. If future trials with larger numbers of participants continue to show such persisting therapeutic effects and a favorable adverse effect profile, psilocybin may garner regulatory approval for use as a medicine in the USA and other nations. If approval is obtained, regulations governing clinical use should closely mirror the screening, preparation, monitoring, and follow-up procedures used in research studies to maximize efficacy and minimize medical and behavioral risks.

Psilocybin in the treatment of addiction is currently at an earlier stage of research, with two recent open-label, pilot 
studies, one for tobacco smoking cessation and one treating alcohol dependence, suggesting potential safety and efficacy. Both of these lines of addiction research are being followed up with larger, randomized studies. If these trials continue to show safety and efficacy, a similar medications development pathway as described for cancer-related psychiatric distress could be anticipated. Although clinical approval for either cancer-related psychiatric distress treatment or addiction treatment do not depend on identification of mechanisms of action, it seems very likely that advancing knowledge of both biological and psychological mechanisms underlying efficacy would lead to advances that improve treatment outcomes.

The US federal funding agencies have yet to support therapeutic psilocybin research. This is understandable given the complex social history surrounding classic psychedelics, and their perceived association with cultural changes that took place in the 1960s and early 1970s. However, as compelling evidence mounts for safety and efficacy, it will be important for government agencies to take a leadership role in supporting cautious and scholarly research on classic psychedelic therapeutics. The recent research with psilocybin, especially evidence of long persisting therapeutic effects that may stem from a single medication administration, suggests that therapy with classic psychedelics may constitute a major new area of medicine that might eventually alleviate suffering across multiple potential disorders.

Acknowledgments Support for effort by the authors has been provided by the Heffter Research Institute and National Institute on Drug Abuse grant R01DA003889. Roland R. Griffiths is on the Board of Directors of the Heffter Research Institute, Santa Fe, NM, USA.

\section{References}

1. Schultes RE. Hallucinogens of plant origin. Science 1969;163:245254.

2. Lowy B. New records of mushroom stones from Guatemala. Mycologia 1971;63:983-993.

3. Schultes RE, Hofmann A. Plants of the gods: their sacred, healing, and hallucinogenic powers. Rochester, VT: Healing Arts Press; 1992.

4. Heffter A. Ueber Pellote. Beitrag zur chemischen und pharmakologischen Kenntnis der Cacteen. NaunynSchmiedebergs Arch Exp Pathol Pharmacol 1898;40:385-429.

5. Perrine DM. Visions of the Night: Western medicine meets peyote 1887-1899. Heffter Rev Psychedel Res 2001;2:6-52.

6. Hofmann A, Ott J. LSD, my problem child. New York: McGrawHill; 1980.

7. Grinspoon L, Bakalar JB. Psychedelic drugs reconsidered. New York: Basic Books; 1979.

8. Grinspoon L. LSD Reconsidered. Sciences 1981;21:20-23.

9. Kast EC, Collins VJ. Study of lysergic acid diethylamide as an analgesic agent. Anesth Analg 1964;43:285-291.

10. Cohen S. LSD and the Anguish of Dying. Harpers Magazine 1965;231:69-72.

11. Kast E. Attenuation of anticipation: a therapeutic use of lysergic acid diethylamide. Psychiatr Q 1967;41:646-657.
12. Kurland AA, Pahnke WN, Unger S, Savage C, Goodman LE. Psychedelic psychotherapy (LSD) in the treatment of the patient with a malignancy. In: Cerletti A, Bové F, editors. The Present Status of Psychotropic Drugs: Pharmacological and Clinical Aspects. Amsterdam: Excerpta Medica 1969:432-434.

13. Pahnke WN, Kurland AA, Goodman LE, Richards WA. LSDassisted psychotherapy with terminal cancer patients. Curr Psychiatr Ther 1969;9:144-152.

14. Richards W, Grof S, Goodman L, Kurland A. LSD-assisted psychotherapy and the human encounter with death. J Transpers Psychol 1972;4:121-150.

15. Kurland AA, Grof S, Pahnke WN, Goodman LE. Psychedelic drug assisted psychotherapy. In: Goldberk IK, Malitz S, Kutscher AH, editors. Patients With Terminal Cancer Psychotheramacological Agents for the Terminally Ill and Bereaved. New York: Columbia University Press; 1973. p. 86-133.

16. Halifax J, Grof S. The Human Encounter with Death. 1st ed. New York: E. P. Dutton; 1977.

17. Richards WA, Rhead JC, DiLeo FB, Yensen R, Kurland AA. The peak experience variable in DPT-assisted psychotherapy with cancer patients. J Psychedel Drugs 1977;9:1-10.

18. Richards WA, Rhead JC, Grof S, Goodman LE, Di Leo F, Rush L. DPT as an adjunct in brief psychotherapy with cancer patients. OMEGA J Death Dying 1980;10:9-26.

19. Grof S. LSD Psychotherapy. 1st ed. Pomona, CA: Hunter House; 1980.

20. Richards WA. Psychedelic drug-assisted psychotherapy with persons suffering from terminal cancer. J Altered States Consciousness 1979;5:309-319.

21. Kurland AA. LSD in the supportive care of the terminally ill cancer patient. J Psychoactive Drugs 1985;17:279-290.

22. Chwelos N, Blewett DB, Smith CM, Hoffer A. Use of d-lysergic acid diethylamide in the treatment of alcoholism. Q J Stud Alcohol 1959;20:577-590.

23. Smart RG, Storm T, Baker EF, Solursh L. A controlled study of lysergide in the treatment of alcoholism: I. The effects on drinking behavior. Q J Stud Alcohol 1966;27:469-482.

24. Hollister LE, Shelton J, Krieger G. A controlled comparison of lysergic acid diethylamide (LSD) and dextroamphetamine in alcoholics. Am J Psychiatry. 1969;125:1352-1357.

25. Ludwig A, Levine J, Stark L, Lazar R. A clinical study of LSD treatment in alcoholism. Am J Psychiatry 1969;126:59-69.

26. Kurland A, Savage C, Pahnke WN, Grof S, Olsson JE. LSD in the treatment of alcoholics. Pharmacopsychiatry 1971;4:83-94.

27. Savage C, McCabe OL. Residential psychedelic (LSD) therapy for the narcotic addict: a controlled study. Arch Gen Psychiatry 1973;28:808-814.

28. Tomsovic M, Edwards RV. Lysergide treatment of schizophrenic and nonschizophrenic alcoholics: a controlled evaluation. Q J Stud Alcohol 1970;31:932-949.

29. Bowen WT, Soskin RA, Chotlos JW. Lysergic acid diethylamide as a variable in the hospital treatment of alcoholism: a follow-up study. J Nerv Mental Dis 1970;150:111-118.

30. Stamets P. Psilocybin mushrooms of the world. Berkeley, CA: Ten Speed Press; 1996.

31. Nichols, DE. Psychedelics. Pharmacol Rev 2016;68:264-355

32. Winter JC, Rice KC, Amorosi DJ, Rabin RA. Psilocybin-induced stimulus control in the rat. Pharmacol Biochem Behav 2007;87: 472-480.

33. Passie T, Seifert J, Schneider U, Emrich HM. The pharmacology of psilocybin. Addict Biol 2002;7:357-364.

34. Fantegrossi WE, Woods JH, Winger G. Transient reinforcing effects of phenylisopropylamine and indolealkylamine hallucinogens in rhesus monkeys. Behav Pharmacol 2004;15:149-157.

35. Griffiths RR, Johnson MW, Richards WA, Richards BD, McCann U, Jesse R. Psilocybin occasioned mystical-type experiences: 
immediate and persisting dose-related effects. Psychopharmacology (Berl) 2011;218:649-665.

36. Johnson MW, Richards WA, Griffiths RR. Human hallucinogen research: guidelines for safety. J Psychopharmacol 2008;22:603620.

37. Johnson MW, Sewell RA, Griffiths RR. Psilocybin dosedependently causes delayed, transient headaches in healthy volunteers. Drug Alcohol Depend 2012;123:132-140.

38. Grob CS, Danforth AL, Chopra GS, et al. Pilot study of psilocybin treatment for anxiety in patients with advanced-stage cancer. Arch Gen Psychiatry 2011;68:71-78.

39. Griffiths RR, Johnson MW, Carducci MA, et al. Psilocybin produces substantial and sustained decreases in depression and anxiety in patients with life-threatening cancer: A randomized double-blind trial. J Psychopharmacol 2016;30:1181-1197.

40. Ross S, Bossis A, Guss J, et al. Rapid and sustained symptom reduction following psilocybin treatment for anxiety and depression in patients with life-threatening cancer: a randomized controlled trial. J Psychopharmacol 2016;30:1165-1180.

41. Gasser P, Holstein D, Michel Y, et al. Safety and efficacy of lysergic acid diethylamide-assisted psychotherapy for anxiety associated with life-threatening diseases. J Nerv Ment Dis 2014;202:513-520.

42. Carhart-Harris RL, Bolstridge M, Rucker J, et al. Psilocybin with psychological support for treatment-resistant depression: an openlabel feasibility study. Lancet Psychiatry 2016;3:619-627

43. Dyck E. 'Hitting highs at rock bottom': LSD treatment for alcoholism, 1950-1970. Soc Hist Med 2006;19:313-329.

44. Krebs TS, Johansen P. Lysergic acid diethylamide (LSD) for alcoholism: meta-analysis of randomized controlled trials. J Psychopharmacol 2012;26:994-1002.

45. Fabregas JM, Gonzlez D, Fondevila S, et al. Assessment of addiction severity among ritual users of ayahuasca. Drug Alcohol Depend 2010;111:257-261.

46. Albaugh BJ, Anderson PO. Peyote in the treatment of alcoholism among American Indians. Am J Psychiatry 1974;131:1247-1250.

47. Bergman RL. Navajo peyote use: its apparent safety. Am J Psychiatry 1971;128:695-699.

48. Blum K, Futterman SFL, Pascarosa P. Peyote, a potential ethnopharmacologic agent for alcoholism and other drug dependencies: possible biochemical rationale. Clin Toxicol 1977;11: 459-472.

49. Calabrese JD. Spiritual healing and human development in the Native American church: toward a cultural psychiatry of peyote. Psychoanal Rev 1997:84:237-255.

50. de Rios MD, Grob CS, Baker JR. Hallucinogens and redemption. J Psychoactive Drugs 2002;34:239-248.

51. Garrity JF. Jesus, peyote, and the holy people: alcohol abuse and the ethos of power in Navajo healing. Med Anthropol Q 2000;14:521542.

52. Halpern JH. The use of hallucinogens in the treatment of addiction. Addict Res 1996;4:177-189.
53. Pascarosa P, Futterman S. Ethnopsychedelic therapy for alcoholics: observations in the peyote ritual of the Native American Church. J Psychedelic Drugs 1976;8:215-221.

54. Prue B. Indigenous supports for recovery from alcoholism and drug abuse: the Native American Church. J Ethn Cult Divers Soc Work 2013;22:271-287.

55. Roy C. Indian peyotists and alcohol. Am J Psychiatry 1973;130: 329-330.

56. Thomas G, Lucas P, Capler NR, Tupper KW, Martin G. Ayahuascaassisted therapy for addiction: Results from a preliminary observational study in Canada. Curr Drug Abuse Rev 2013;6:30-42.

57. Lattin D. Distilled Spirits: Getting High, then Sober, with a Famous Writer, a Forgotten Philosopher, and a Hopeless Drunk. Berkeley, CA: University of California Press; 2012.

58. Johnson MW, Garcia-Romeu A, Cosimano MP, Griffiths RR. Pilot study of the 5-HT2AR agonist psilocybin in the treatment of tobacco addiction. J Psychopharmacol 2014;28:983-992.

59. Johnson MW, Garcia-Romeu A, Griffiths RR. Long-term follow-up of psilocybin-facilitated smoking cessation. Am J Drug Alcohol Abuse 2017;43:55-60.

60. Garcia-Romeu A, Griffiths R R, Johnson M. W. Psilocybinoccasioned mystical experiences in the treatment of tobacco addiction. Curr Drug Abuse Rev 2014;7:157-164.

61. Johnson MW, Garcia-Romeu A, Johnson PS, Griffiths RR. An online survey of tobacco smoking cessation associated with naturalistic psychedelic use. J Psychopharmacol 2017 Jan 1.

62. Bogenschutz MP, Forcehimes AA, Pommy JA, Wilcox CE, Barbosa P, Strassman RJ. Psilocybin-assisted treatment for alcohol dependence: a proof-of-concept study. J Psychopharmacol 2015;29:289-299.

63. Moreno FA, Wiegand CB, Taitano EK, Delgado PL. Safety, tolerability, and efficacy of psilocybin in 9 patients with obsessivecompulsive disorder. J Clin Psychiatry 2006;67:1735-1740.

64. Sewell RA, Halpern JH, Pope HG. Response of cluster headache to psilocybin and LSD. Neurology 2006;66:1920-1922.

65. Schindler EA, Gottschalk CH, Weil MJ, Shapiro RE, Wright DA, Sewell RA. Indoleamine hallucinogens in cluster headache: results of the Clusterbusters medication use survey. J Psychoact Drugs 2015;47:372-381.

66. Karst M, Halpern JH, Bernateck M, Passie T. The non-hallucinogen 2-bromo-lysergic acid diethylamide as preventative treatment for cluster headache: an open, non-randomized case series. Cephalalgia 2010;30:1140-1144.

67. Carhart-Harris RL, Erritzoe D, Williams T, et al. Neural correlates of the psychedelic state as determined by fMRI studies with psilocybin. Proc Natl Acad Sci 2012;109:2138-2143.

68. Nichols DE, Johnson MW, Nichols CD. Psychedelics as medicines: an emerging new paradigm. Clin Pharmacol Ther 2017;101:209219. 